\title{
Stereotactic Body Radiation Therapy vs.
}

Radiofrequency Ablation for Single Small

Hepatocellular Carcinoma: A Propensity-score

Matching Analysis of Their Impact on Liver Function and Clinical Outcomes

Masayuki Ueno ( $\sim$ mu13951@kchnet.or.jp )

Kurashiki Central Hospital

Hiroyuki Takabatake

Kurashiki Central Hospital

Satoshi Itasaka

Kurashiki Central Hospital

Takahisa Kayahara

Kurashiki Central Hospital

Youichi Morimoto

Kurashiki Central Hospital

Hiroshi Yamamoto

Kurashiki Central Hospital

Motowo Mizuno

Kurashiki Central Hospital

\section{Research Article}

Keywords: hepatocellular carcinoma, stereotactic body radiotherapy, radiofrequency ablation, liver function, survival, propensity-scores

Posted Date: February 24th, 2021

DOI: https://doi.org/10.21203/rs.3.rs-228801/v1

License: (c) (1) This work is licensed under a Creative Commons Attribution 4.0 International License.

Read Full License 


\section{Abstract}

Background: Stereotactic body radiation therapy (SBRT) has high efficacy for early-stage hepatocellular carcinoma (HCC) and is expected as an accepted alternative to radiofrequency ablation (RFA). However, SBRT for HCC may cause subacute liver injury leading to negative clinical outcomes. In this study, we compared changes of liver function and prognosis after SBRT or RFA in patients with single, small HCC by using a propensity-score matching analysis.

Methods: We reviewed medical records of 140 patients with single $\leq 3 \mathrm{~cm}$ HCC treated with SBRT or RFA at Kurashiki Central Hospital between January 2014 and February 2019. Changes of albumin-bilirubin (ALBI) score, local recurrence, and overall survival were compared between the propensity-score matched groups (31 patients treated with SBRT and 62 treated with RFA).

Results: The ALBI score increased modestly but significantly after SBRT, while it was unchanged in the RFA group; the intergroup difference was statistically significant $(p=0.004)$. No local recurrence of tumor was identified in the SBRT group, whereas the cumulative recurrence incidence was $9.7 \%$ in the RFA group $(p=0.023)$. Overall survival was not significantly different between the two groups (hazard ratio 1.32, $95 \%$ confidence interval $0.60-2.89, p=0.401$ ).

Conclusions: SBRT had modestly negative impact on liver function but better local control of HCC than did RFA. Although long-term follow-up of liver function is necessary with SBRT treatment especially in patients with impaired liver function, SBRT is a satisfactory alternative to RFA for treatment of single, small HCC.

\section{Background}

Hepatocellular carcinoma (HCC) is the fourth most common cause of cancer-related death worldwide [1]. Curative treatment at an early stage is a cornerstone for improving the outcome of HCC [2]. In the current guidelines, surgical resection, radiofrequency ablation (RFA), or liver transplantation is recommended for treatment of early-stage HCC [3]. However, each treatment has drawbacks: Surgical resection is invasive and may cause postoperative morbidity and liver failure [4]; the curability with RFA may be limited by tumor size and its location $[5,6]$; and, because of the scarcity of donor organs, liver transplantation is rarely performed in Asian countries [7].

Remarkable technological advances in radiotherapy for HCC have been made in recent years [8]. Stereotactic body radiation therapy (SBRT) uses hypo-fractionated irradiation with a very high dose per fraction, which enables satisfactory therapeutic effects with lower toxicity [8]. The local control rate of SBRT for early-stage HCC has been reported to be $87-100 \%$ [9-11]. SBRT is expected to overcome the drawbacks of surgical resection and RFA because it is less invasive than resection, and it is less influenced by tumor size and location than is RFA [6]. 
However, important issues with SBRT remain to be clarified. First, SBRT can cause radiation-induced liver disease (RILD) [12,13]. Thanks to modern radiotherapy techniques, classic RILD (i.e., hepatomegaly, ascites, and elevated liver enzymes) is rare in the current era [14]. Nonetheless, non-classic RILD, which also is associated with worse prognosis, sometimes occurs [13], and the long-term impact of SBRT on liver function is uncertain. Second, survival outcomes with SBRT are variable; a multi-center study reported better overall survival with SBRT than with RFA $[6,15]$, whereas an analysis using the National Cancer Database reported an opposite result $[16,17]$. In this study, using a propensity-score matching analysis, we compared the long-term impact on liver function and clinical outcomes of patients with single, small HCC treated with SBRT or RFA.

\section{Methods}

\section{Patients}

We conducted a retrospective study by reviewing medical records of 641 patients with HCC treated at Kurashiki Central Hospital between January 2014 and February 2019. The inclusion criteria for this study were 1) a single HCC of $3 \mathrm{~cm}$ or smaller; 2) diagnosis established by histology or contrast-enhanced imaging; 3 ) treatment with SBRT or RFA; and 4) patients age 20 years old or older. The exclusion criteria were 1) macrovascular invasion or extrahepatic metastasis; 2) Child-Pugh score 10 or higher; and 3) refusal to join in the study. One hundred forty patients were included in this study; 44 were treated with SBRT and 96 with RFA. In our institution, RFA was the first-choice therapy for single, small HCC, and SBRT was chosen when RFA was considered inappropriate (e.g., local recurrence after RFA and tumors adjacent to the vessels or liver surface). All procedures were performed in accordance with the Helsinki Declaration of 1975 , as revised in 2008. This study was approved by the Medical Ethics Committee of Kurashiki Central Hospital (no. 2948). Informed consent was obtained from all patients included in the study in an opt-out protocol.

\section{SBRT procedure}

Patients were immobilized with use of an individually shaped vacuum pillow and underwent a 4dimensional (4D) computed tomography (CT) scan. The gross tumor volume was delineated with the assistance of contrast-enhanced CT scans and MRI (magnetic resonance imaging). The 4D CT was used to help create an internal target volume to compensate for respiratory motion. The planning target volume included the internal target volume plus a 3- to 5-mm expansion. A stereotactic, multiarc, dynamic, conformal radiation procedure or volumetric modulated arc therapy was performed by use of TrueBeam or Clinac IX (Varian Medical Systems, Palo Alto, CA), with respiratory gating or breath hold. Target alignment and setup accuracy were ensured through use of implanted fiducial markers (Gold Anchor $^{T M}$ (Naslund Medical AB, Huddinge, Sweden) or VISICOIL ${ }^{T M}$ (IZI Medical Products, Baltimore, USA)). A total dose of $40 \mathrm{~Gy}$ was delivered in five fractions over 5-9 days on either an inpatient or outpatient basis.

\section{RFA procedure}


RFA was performed with real-time ultrasonographic guidance on an inpatient basis. We used Cool-tip ${ }^{\text {TM }}$ RF Ablation System E Series (Covidien, Mansfield, USA) and tried to create an ablative margin of at least 5 mm surrounding the tumor. Contrast-enhanced CT or nonenhanced MRI was performed on the day after RFA; if ablation was incomplete, an additional session was conducted during the same hospital stay.

\section{Follow-up}

After treatment, patients were assessed every 3 months with laboratory and radiological examination. Contrast-enhanced CT or MRI was performed at least every 6 months unless patients had contraindications to contrast administration. Post-treatment response was assessed according to the Response Evaluation Criteria in Cancer of the Liver [18]. If HCC recurred, appropriate treatment was provided based on the clinical guideline [3].

\section{Statistical analysis}

Categorical variables were expressed as numbers (percentages) and continuous variables were expressed as median [interquartile range] or mean \pm standard deviation. To compare differences in patients' characteristics between the two groups, Fisher's exact test and the Mann-Whitney U test were used for categorical values and continuous values, respectively. Longitudinal changes of albumin-bilirubin (ALBI) score, local recurrence, and overall survival were compared between the propensity-score matched groups. Time to local recurrence was defined as the time from SBRT or RFA to radiologic diagnosis of local recurrence. Overall survival was defined as the time from SBRT or RFA to all-cause death, with living patients censored on the date of last follow-up. Patients in the SBRT group were matched at a 1:2 ratio with patients treated with RFA. The variables included in the propensity-score model were age, tumor size, performance status, baseline ALBI score, baseline Child-Pugh score, and the number of previous treatments for HCC. In each treatment group, ALBI scores at 12 months after treatment were compared with the baseline values by using the Wilcoxon rank sum test. To analyze the intergroup difference of the longitudinal change of ALBI scores, repeated analysis of variance was employed. Kaplan-Meier analysis, the log-rank test, and the Cox proportional hazards models were used for analysis of local recurrence and overall survival. A two-tailed $p$ value of $<0.05$ was considered statistically significant. All statistical analyses were performed with R (version 3.5.1, R Foundation) and JMP (version 12.2, SAS Institute Japan).

\section{Results}

\section{Baseline characteristics}

Among the 140 patients, 101 (72.1\%) were male, and the median age was 75 [67.3-81] years. Most patients $(80.7 \%)$ had well-compensated liver function (Child-Pugh class A). Median tumor size was 13 $\mathrm{mm}$ [10-20 mm]. Before propensity-score matching (Table 1), patients in the SBRT group had significantly higher age, worse performance status, higher proportion of stage A tumors, and more patients having had previous treatment than did those in the RFA group. In addition, SBRT was more 
frequently performed as salvage therapy for recurrence after RFA or transcatheter arterial chemoembolization (TACE). After the matching (Table 2), 31 patients treated with SBRT were compared with 62 patients treated with RFA. ALBI scores and other baseline characteristics were well balanced between the two groups, but patients with HCV infection were more numerous in the RFA group (41/62, $66.1 \%$ vs. $13 / 62,41.9 \% ; p=0.044)$, and the frequency of salvage therapy was significantly higher in the SBRT group $(8 / 23,25.8 \%$ vs. $1 / 16,1.6 \%, p<0.001)$. 
Table 1

Patients' baseline characteristics before propensity score matching

\begin{tabular}{|c|c|c|c|}
\hline & $\begin{array}{l}\text { SBRT group } \\
(n=44)\end{array}$ & $\begin{array}{l}\text { RFA group } \\
(n=96)\end{array}$ & $p$ value \\
\hline Age (years) & 78 [70-82] & 73 [67-79] & 0.020 \\
\hline Sex (male/female) & $32 / 12$ & $69 / 27$ & 1.000 \\
\hline Etiology & & & 0.170 \\
\hline HBV & $8(18.2 \%)$ & $12(12.5 \%)$ & \\
\hline $\mathrm{HCV}$ & $22(50.0 \%)$ & $64(66.7 \%)$ & \\
\hline Others & $14(31.8 \%)$ & $20(20.8 \%)$ & \\
\hline Tumor size $(\mathrm{mm})$ & 14 [10-23] & $13[10-17]$ & 0.189 \\
\hline Tumor location, segment & & & 0.222 \\
\hline $1 / 2 / 3 / 4$ & $0 / 1 / 2 / 7$ & $1 / 3 / 6 / 16$ & \\
\hline $5 / 6 / 7 / 8$ & $4 / 7 / 10 / 13$ & $17 / 16 / 6 / 31$ & \\
\hline BCLC staging $(0 / A)$ & $27 / 17$ & $76 / 20$ & 0.044 \\
\hline Total bilirubin (mg/dL) & $0.6[0.4-0.9]$ & $0.6[0.5-0.9]$ & 0.637 \\
\hline Albumin (g/dL) & $3.5[3.2-3.8]$ & $3.5[3.1-3.9]$ & 0.597 \\
\hline ALBI score & $-2.40[-2.51--1.93]$ & $-2.33[-2.65--1.97]$ & 0.760 \\
\hline Child-Pugh score $(5 / 6 / 7 / 8 / 9)$ & $19 / 18 / 6 / 1 / 0$ & 43 / 33 / 12 / 5 / 3 & 0.666 \\
\hline Performance status $(0 / 1)$ & $32 / 12$ & $85 / 11$ & 0.036 \\
\hline \multicolumn{4}{|l|}{ Prior treatment } \\
\hline Surgery & $21(47.7 \%)$ & 37 (38.5\%) & 0.401 \\
\hline RFA & $28(63.6 \%)$ & $55(57.3 \%)$ & 0.600 \\
\hline TACE & $32(72.7 \%)$ & $44(45.8 \%)$ & 0.005 \\
\hline Others & $10(22.7 \%)$ & 3 (3.1\%) & $<0.001$ \\
\hline Number of prior treatments & $3.5[2-6]$ & $2[0-4]$ & 0.006 \\
\hline
\end{tabular}

SBRT, stereotactic body radiation therapy; RFA, radiofrequency ablation; HBV, hepatitis B virus; $\mathrm{HCV}$, hepatitis C virus; BCLC, Barcelona Clinic liver cancer; ALBI, albumin-bilirubin; TACE, transcatheter arterial chemoembolization.

*recurrence after RFA or TACE. 


\begin{tabular}{|llll|}
\hline & $\begin{array}{l}\text { SBRT group } \\
(\mathbf{n = 4 4 )}\end{array}$ & $\begin{array}{l}\text { RFA group } \\
(\mathbf{n = 9 6 )}\end{array}$ & $p$ value \\
\hline Treatment setting (salvage*/not salvage) & $11 / 33$ & $1 / 95$ & $<0.001$ \\
\hline $\begin{array}{l}\text { SBRT, stereotactic body radiation therapy; RFA, radiofrequency ablation; HBV, hepatitis B virus; HCV, } \\
\text { hepatitis C virus; BCLC, Barcelona Clinic liver cancer; ALBI, albumin-bilirubin; TACE, transcatheter } \\
\text { arterial chemoembolization. }\end{array}$ & & \\
\hline *recurrence after RFA or TACE. & & \\
\hline
\end{tabular}


Table 2

Patients' baseline characteristics after propensity score matching

\begin{tabular}{|c|c|c|c|c|}
\hline & $\begin{array}{l}\text { SBRT group } \\
(n=31)\end{array}$ & $\begin{array}{l}\text { RFA group } \\
(n=62)\end{array}$ & SMD & $p$ value \\
\hline Age $^{*}$, years & $75.2 \pm 9.1$ & $75.0 \pm 7.5$ & 0.025 & 0.907 \\
\hline Sex (male/female) & $24 / 7$ & $43 / 19$ & 0.183 & 0.471 \\
\hline \multicolumn{5}{|l|}{ Etiology } \\
\hline HBV & $8 / 23$ & $7 / 55$ & 0.380 & 0.132 \\
\hline $\mathrm{HCV}$ & $13 / 18$ & $41 / 21$ & 0.500 & 0.044 \\
\hline Tumor size ${ }^{\star}(\mathrm{mm})$ & $14.7 \pm 6.4$ & $15.0 \pm 5.5$ & 0.043 & 0.842 \\
\hline Tumor location (right lobe/left lobe) & $25 / 6$ & $48 / 14$ & 0.079 & 0.795 \\
\hline BCLC staging (0/A) & $22 / 9$ & $46 / 16$ & 0.072 & 0.806 \\
\hline ALBI score* & $-2.32 \pm 0.45$ & $-2.28 \pm 0.46$ & 0.088 & 0.692 \\
\hline Child-Pugh score ${ }^{*}$ & $5.74 \pm 0.81$ & $5.82 \pm 1.05$ & 0.086 & 0.709 \\
\hline Performance status $(0 / 1)$ & $26 / 5$ & $51 / 11$ & 0.043 & 1.000 \\
\hline Number of prior treatments ${ }^{*}$ & $3.9 \pm 3.1$ & $3.7 \pm 3.4$ & 0.065 & 0.774 \\
\hline Surgery & $0.52 \pm 0.63$ & $0.55 \pm 0.69$ & 0.049 & 0.828 \\
\hline RFA & $1.39 \pm 1.63$ & $1.55 \pm 1.60$ & 0.100 & 0.649 \\
\hline TACE & $1.68 \pm 1.47$ & $1.40 \pm 1.58$ & 0.180 & 0.422 \\
\hline Treatment setting (salvage/ not salvage) & $8 / 23$ & $1 / 61$ & 0.751 & $<0.001$ \\
\hline \multicolumn{5}{|c|}{$\begin{array}{l}\text { SBRT, stereotactic body radiation therapy; RFA, radiofrequency ablation; SMD, standardized mean } \\
\text { difference; HBV, hepatitis B virus; HCV, hepatitis C virus; BCLC, Barcelona Clinic liver cancer; ALBI, } \\
\text { albumin-bilirubin; TACE, transcatheter arterial chemoembolization }\end{array}$} \\
\hline \multicolumn{5}{|c|}{ *variables included in the propensity score model } \\
\hline
\end{tabular}

\section{Longitudinal changes of ALBI score}

Figure 1 illustrates the longitudinal ALBI scores in the two treatment groups. In the SBRT group, the ALBI score gradually increased (i.e., liver function got worse) between the baseline and 12 months after treatment $(-2.32 \pm 0.45$ vs. $-2.17 \pm 0.61, p<0.001)$ (Fig. $1 \mathrm{~A})$. In contrast, the ALBI score was unchanged in the RFA group ( $-2.28 \pm 0.46$ at the baseline vs. $-2.30 \pm 0.55$ at 12 months after treatment, $p=0.839)$ (Fig. 1B). Table 3 presents the numerical values for the longitudinal changes in ALBI values for the SBRT and RFA groups; the intergroup difference of the longitudinal changes of ALBI scores was statistically 
significant in the propensity-score matched cohort $(p=0.004)$ and in the whole cohort $(p=0.034)$. Figure 2 illustrates the subgroup analysis stratified by baseline modified ALBI (mALBI) grade [19]; whereas ALBI scores increased after SBRT in patients who had reduced liver function (mALBI grade 2a, $p$ $=0.007$; and $2 b, p=0.041$ ) at baseline, scores did not increase in the patients who had good ALBI score (mALBI grade 1, $p=0.285$ ) at baseline. During the 12 months after therapy, intrahepatic recurrence of HCC including local recurrence was identified in 9 patients in the SBRT group (29.0\%) and 19 patients in the RFA group (30.6\%), a statistically insignificant difference $(p=1.000)$. This result is evident that recurrence of HCC was not responsible for differences in longitudinal changes of ALBI scores in the two groups.

Table 3

Longitudinal change of ALBI score after treatment

\begin{tabular}{|lccc|}
\hline & SBRT group & RFA group & $P_{\text {interaction }}{ }^{*}$ \\
\hline Matched cohort $(\mathrm{n}=93)$ & & & 0.004 \\
\hline Baseline & $-2.32 \pm 0.45$ & $-2.28 \pm 0.46$ & \\
\hline 3 months & $-2.33 \pm 0.48$ & $-2.29 \pm 0.43$ & \\
\hline 6 months & $-2.28 \pm 0.47$ & $-2.31 \pm 0.57$ & \\
\hline 9 months & $-2.15 \pm 0.56$ & $-2.36 \pm 0.48$ & \\
\hline 12 months & $-2.17 \pm 0.61$ & $-2.30 \pm 0.55$ & \\
\hline Cohort before the matching $(n=140)$ & & & \\
\hline Baseline & $-2.26 \pm 0.52$ & $-2.29 \pm 0.49$ & \\
\hline 3 months & $-2.28 \pm 0.55$ & $-2.30 \pm 0.47$ & \\
\hline 6 months & $-2.17 \pm 0.65$ & $-2.32 \pm 0.57$ & \\
\hline 9 months & $-2.13 \pm 0.65$ & $-2.34 \pm 0.54$ & \\
\hline 12 months & $-2.16 \pm 0.68$ & $-2.30 \pm 0.56$ & \\
\hline $\begin{array}{l}\text { SBRT, stereotactic body radiation therapy; RFA, radiofrequency ablation; HCC, hepatocellular } \\
\text { carcinoma }\end{array}$ & & \\
\hline *Repeated measures analysis of variance & & & \\
\hline
\end{tabular}

\section{Local recurrence and overall survival}

Figure $3 \mathrm{~A}$ illustrates that during the follow-up periods of up to 63.3 months (median, 23.4 months) in the SBRT group and up to 76.6 months (median, 27.1 months) in the RFA group, no local recurrence of HCC was identified in the SBRT group, whereas 6 cases were identified in the RFA group, a cumulative incidence of $9.7 \%(p=0.024)$. Figure 3B illustrates that during the follow-up periods, $10(32.3 \%)$ patients 
in the SBRT group and 17 (27.4\%) patients in the RFA group died, a statistically insignificant difference (hazard ratio $1.32,95 \%$ confidence interval $0.60-2.89, p=0.401$ ).

\section{Discussion}

In this study of therapy for single, small HCC, SBRT had significantly worse impact on liver function, assessed by ALBI score, but achieved better local control than did RFA. Despite these differences, overall survival was similar between patients treated with the two procedures. To our best knowledge, this is the first study that compared, with propensity-score matching analysis, the long-term effects of SBRT and RFA on liver function.

In studies that have used Child-Pugh score for assessment of liver toxicity, the changes of liver function after treatment were not significantly different between SBRT- and RFA-treated patients $[15,20]$. We used ALBI score for assessment of liver function - which is reportedly a better predictor of the prognosis of HCC than is Child-Pugh score [21] - to analyze the liver function as a continuous variable. Thus, we could analyze the delicate changes of liver function, which disclosed the differences between SBRT and RFA treatment.

The increases in ALBI score became noticeable more than 6 months after SBRT. This long interlude can be explained by the fact that, after SBRT, blood flow decreases and the necrotic parenchyma expands over several months $[22,23]$. We also found that increase of ALBI scores was associated with abnormal liver function (mALBI grade $2 a$ and $2 b$ ) at baseline. This observation is in accordance with reports that worse liver function at baseline was associated with higher risk of RILD [24-26]. Thus, careful follow-up of liver function should be continued for at least 1 year after SBRT, especially in patients with known impaired liver function.

Although liver toxicity, defined by increased ALBI score, was associated with SBRT more than with RFA, the degree was mild and not critical. Moreover, it should be appreciated that the excellent local control rate and the similar overall survival with SBRT was achieved despite the procedure being mostly performed for tumors that were difficult to completely ablate with RFA. Other studies also have found better local control of HCC with SBRT than with RFA, especially for perivascular tumors $[6,15,20]$. Since complete ablation is associated with better prognosis [2], the excellent local control with SBRT may also be beneficial.In contrast to our findings, an analysis of the National Cancer Database in the United State found SBRT inferior to RFA in survival benefit [14]. However, that study did not include important variables, such as liver function, performance status, and treatment history for HCC, as matching factors. Other studies that balanced baseline liver function and previous treatment history found that survival outcomes with SBRT were similar to $[15,28]$ or even superior to outcomes with RFA [6]. We also included the numbers of previous treatment and liver function in the propensity-score model and found that overall survival with SBRT was like that with RFA. Taken together, our findings suggest that SBRT is a promising treatment for early-stage HCC, especially for HCCs that are difficult to treat with RFA, despite its modest adverse effect on liver function. 
The adverse effect on liver function in our patients treated with SBRT occurred despite our cautious use of that modality. Patients were treated with a total dose of 40Gy, TACE was not combined, and fiducial gold marker was used to compensate for respiratory liver motion. In other institution, doses higher than 40Gy together with TACE have been used [29,30]. The results of our subgroup analysis stratified by baseline liver function suggest that lower radiation dose (35Gy) may be appropriate for patients with impaired liver function [15].

Limitations of our study are these: 1) It is a retrospective study, thus inherently flawed by selection and indication bias; though we performed propensity-score matching, unidentified confounding factors could have existed. A prospective study (UMIN000036081) is ongoing in our institution to reassess the present results. 2) Dose and fractions of radiation used for HCC may not have been optimal. Although giving 40 Gy in five fractions is a commonly accepted protocol for treating $\operatorname{HCC}[15,30]$, the issue needs further study.

\section{Conclusions}

SBRT is a promising alternative to RFA for treatment of single, small HCC despite need for long-term monitoring of liver function, especially in patients with pretreatment impaired liver function.

\section{Abbreviations}

- 4D: 4-dimensional

- ALBI: albumin-bilirubin

- HCC: hepatocellular carcinoma

- RFA: radiofrequency ablation

- RILD: radiation-induced liver disease

- SBRT: stereotactic body radiation therapy

- TACE: transcatheter arterial chemoembolization

\section{Declarations}

\section{Ethics approval and consent to participate:}

This study was approved by the Medical Ethics Committee of Kurashiki Central Hospital (no. 2948). Informed consent was obtained from all patients included in the study in an opt-out protocol.

\section{Consent for publication:}

Not applicable 


\section{Availability of data and materials:}

The datasets used and/or analysed during the current study are available from the corresponding author on reasonable request.

\section{Competing interests:}

The authors declare that they have no competing interests.

\section{Funding:}

None

\section{Authors' contributions:}

MUand HT designed the study. MU collected the data. All authors analyzed and interpreted the data. MU and SI wrote the draft of the manuscript and all other authors revised it. Final version of the manuscript was approved by all authors. All authors agreed to be accountable for all aspects of the work.

\section{Acknowledgments:}

We are grateful to A. Takeda for helpful discussions and comments on the manuscript. We also thank W. R. Brown for the English language review.

\section{References}

1. Yang JD, Hainaut P, Gores GJ, Amadou A, Plymoth A, Roberts LR. A global view of hepatocellular carcinoma: trends, risk, prevention and management. Nat Rev Gastroenterol Hepatol. 2019;16:589604.

2. Kudo M, Izumi N, Sakamoto M, Matsuyama Y, Ichida T, Nakashima O, et al. Survival analysis over 28 years of 173,378 patients with hepatocellular carcinoma in Japan. Liver Cancer. 2016;5:190-7.

3. Korean Liver Cancer Association (KLCA); National Cancer Center (NCC), Goyang, Korea. 2018 Korean Liver Cancer Association-National Cancer Center Korea practice guidelines for the management of hepatocellular carcinoma. Korean J Radiol. 2019;20:1042-113.

4. Peng ZW, Lin XJ, Zhang YJ, Liang HH, Guo RP, Shi M, et al. Radiofrequency ablation versus hepatic resection for the treatment of hepatocellular carcinomas $2 \mathrm{~cm}$ or smaller: a retrospective comparative study. Radiology. 2012;262:1022-33. 
5. Lee S, Kang TW, Cha DI, Song KD, Lee MW, Rhim H, et al. Radiofrequency ablation vs. surgery for perivascular hepatocellular carcinoma: Propensity score analyses of long-term outcomes. J Hepatol. 2018;69:70-8.

6. Kim N, Cheng J, Jung I, Liang J, Shih YL, Huang WY, et al. Stereotactic body radiation therapy vs. radiofrequency ablation in Asian patients with hepatocellular carcinoma. J Hepatol. 2020;73:121-9.

7. Chan SC. Liver transplantation for hepatocellular carcinoma. Liver Cancer. 2013;2:338-44.

8. Qiu B, Aili A, Xue L, Jiang P, Wang J. Advances in radiobiology of stereotactic ablative radiotherapy. Front Oncol. 2020;10:1165.

9. Cárdenes HR, Price TR, Perkins SM, Maluccio M, Kwo P, Breen TE, et al. Phase I feasibility trial of stereotactic body radiation therapy for primary hepatocellular carcinoma. Clin Transl Oncol. 2010;12:218-25.

10. Bujold A, Massey CA, Kim JJ, Brierley J, Cho C, Wong RK, et al. Sequential phase I and II trials of stereotactic body radiotherapy for locally advanced hepatocellular carcinoma. J Clin Oncol. 2013;31:1631-9.

11. Kim JW, Seong J, Lee IJ, Woo JY, Han KH. Phase I dose escalation study of helical intensitymodulated radiotherapy-based stereotactic body radiotherapy for hepatocellular carcinoma. Oncotarget. 2016;7:40756-66.

12. Pan CC, Kavanagh BD, Dawson LA, Li XA, Das SK, Miften M, et al. Radiation-associated liver injury. Int J Radiat Oncol Biol Phys. 2010;76:S94-100.

13. Sanuki N, Takeda A, Oku Y, Eriguchi T, Nishimura S, Aoki Y, et al. Influence of liver toxicities on prognosis after stereotactic body radiation therapy for hepatocellular carcinoma. Hepatol Res. 2015;45:540-7.

14. Koay EJ, Owen D, Das P. Radiation-induced liver disease and modern radiotherapy. Semin Radiat Oncol. 2018;28:321-31.

15. Hara K, Takeda A, Tsurugai Y, Saigusa Y, Sanuki N, Eriguchi T, et al. Radiotherapy for hepatocellular carcinoma results in comparable survival to radiofrequency ablation: A propensity score analysis. Hepatology. 2019;69:2533-45.

16. Rajyaguru DJ, Borgert AJ, Smith AL, Thomes RM, Conway PD, Halfdanarson TR, et al. Radiofrequency ablation versus stereotactic body radiotherapy for localized hepatocellular carcinoma in nonsurgically managed patients: Analysis of the National Cancer Database. J Clin Oncol. 2018;36:600-8.

17. Kudo M, Ikeda M, Ueshima K, Sakamoto M, Shiina S, Tateishi R, et al. Response Evaluation Criteria in Cancer of the Liver version 5 (RECICL 2019 revised version). Hepatol Res. 2019;49:981-9.

18. Berger NG, Tanious MN, Hammad AY, Miura JT, Mogal H, Clarke CN, et al. External radiation or ablation for solitary hepatocellular carcinoma: A survival analysis of the SEER database. J Surg Oncol. 2017;116:307-12.

19. Hiraoka A, Michitaka K, Kumada T, Izumi N, Kadoya M, Kokudo N, et al. Validation and potential of albumin-bilirubin grade and prognostication in a nationwide survey of 46,681 hepatocellular 
carcinoma patients in Japan: the need for a more detailed evaluation of hepatic function. Liver Cancer. 2017;6:325-36.

20. Kim N, Kim HJ, Won JY, Kim DY, Han KH, Jung I, et al. Retrospective analysis of stereotactic body radiation therapy efficacy over radiofrequency ablation for hepatocellular carcinoma. Radiother Oncol. 2019;131:81-7.

21. Peng $Y$, Wei Q, He Y, Xie Q, Liang Y, Zhang L, et al. ALBI versus child-pugh in predicting outcome of patients with HCC: A systematic review. Expert Rev Gastroenterol Hepatol. 2020;14:383-400.

22. Price TR, Perkins SM, Sandrasegaran K, Henderson MA, Maluccio MA, Zook JE, et al. Evaluation of response after stereotactic body radiotherapy for hepatocellular carcinoma. Cancer. 2012;118:31918.

23. Mendiratta-Lala M, Gu E, Owen D, Cuneo KC, Bazzi L, Lawrence TS, et al. Imaging findings within the first 12 months of hepatocellular carcinoma treated with stereotactic body radiation therapy. Int $J$ Radiat Oncol Biol Phys. 2018;102:1063-9.

24. Jung J, Yoon SM, Kim SY, Cho B, Park JH, Kim SS, et al. Radiation-induced liver disease after stereotactic body radiotherapy for small hepatocellular carcinoma: clinical and dose-volumetric parameters. Radiat Oncol. 2013;8:249.

25. Huang Y, Chen SW, Fan CC, Ting LL, Kuo CC, Chiou JF. Clinical parameters for predicting radiationinduced liver disease after intrahepatic reirradiation for hepatocellular carcinoma. Radiat Oncol. 2016;11:89.

26. Jun BG, Kim YD, Cheon GJ, Kim ES, Jwa E, Kim SG, et al. Clinical significance of radiation-induced liver disease after stereotactic body radiation therapy for hepatocellular carcinoma. Korean J Intern Med. 2018;33:1093-102.

27. Sparchez Z, Mocan T, Radu P, Mocan LP, Sparchez M, Leucuta DC, et al. Prognostic factors after percutaneous radiofrequency ablation in the treatment of hepatocellular carcinoma. Impact of incomplete ablation on recurrence and overall survival rates. J Gastrointestin Liver Dis. 2018;27:399407.

28. Wahl DR, Stenmark MH, Tao Y, Pollom EL, Caoili EM, Lawrence TS, et al. Outcomes after stereotactic body radiotherapy or radiofrequency ablation for hepatocellular carcinoma. J Clin Oncol. 2016;34:452-9.

29. Baumann BC, Wei J, Plastaras JP, Lukens JN, Damjanov N, Hoteit M, et al. Stereotactic body radiation therapy (SBRT) for hepatocellular carcinoma: High rates of local control with low toxicity. Am J Clin Oncol. 2018;41:1118-24.

30. Kimura T, Takeda A, Sanuki N, Ariyoshi K, Yamaguchi T, Imagumbai T, et al. Multicenter prospective study of stereotactic body radiotherapy for previously untreated solitary primary hepatocellular carcinoma: The STRSPH study. Hepatol Res. 2020; doi:10.1111/hepr.13595.

\section{Figures}


Figure. 1A

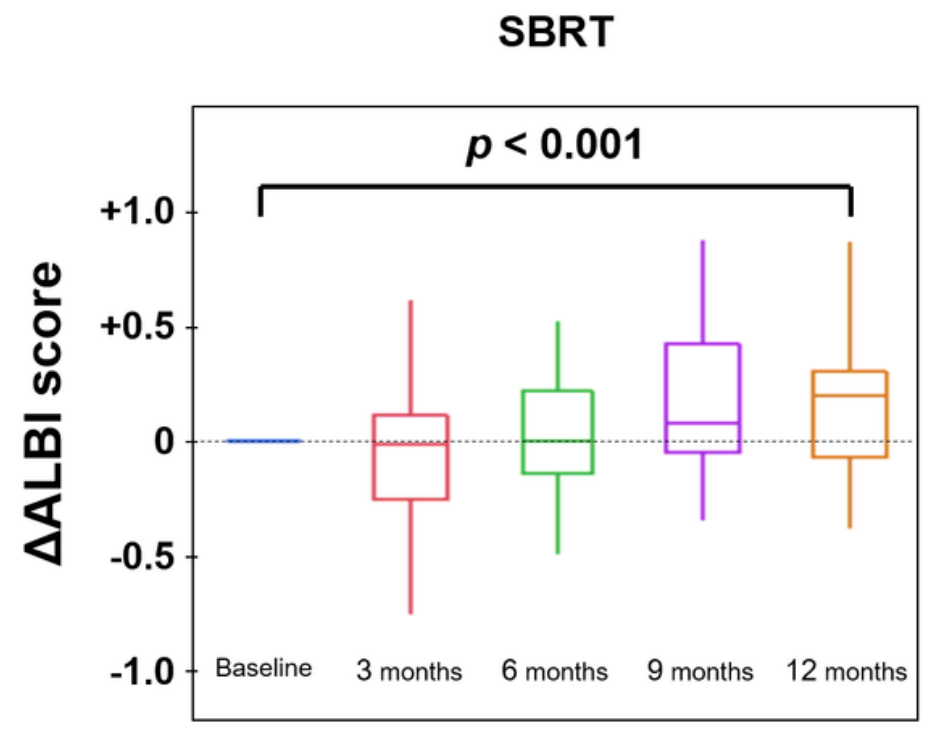

Figure. 1B

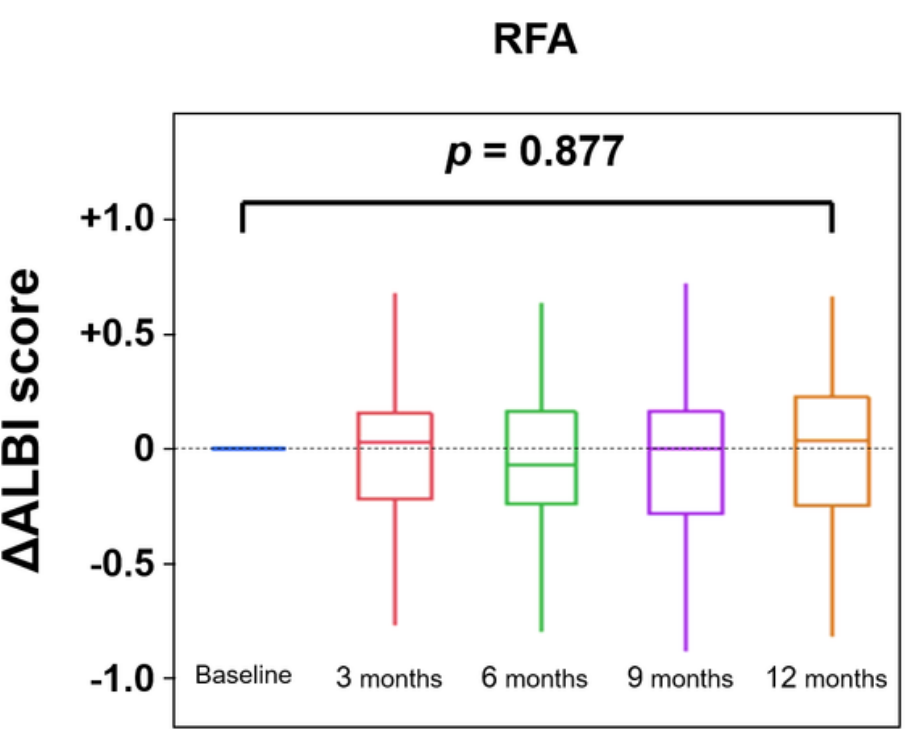

\section{Figure 1}

Longitudinal changes of ALBI score in the SBRT group (A) and RFA group (B). Differences of ALBI scores from baseline ( $\triangle \mathrm{ALBI}$ scores) are shown in the figure. Vertical lines represent the ranges and box contains values between 25th and 75th percentiles (central line, median). ALBI scores at 12 months in the SBRT group were significantly increased from baseline, whereas scores were unchanged in the RFA group.

Figure. 2

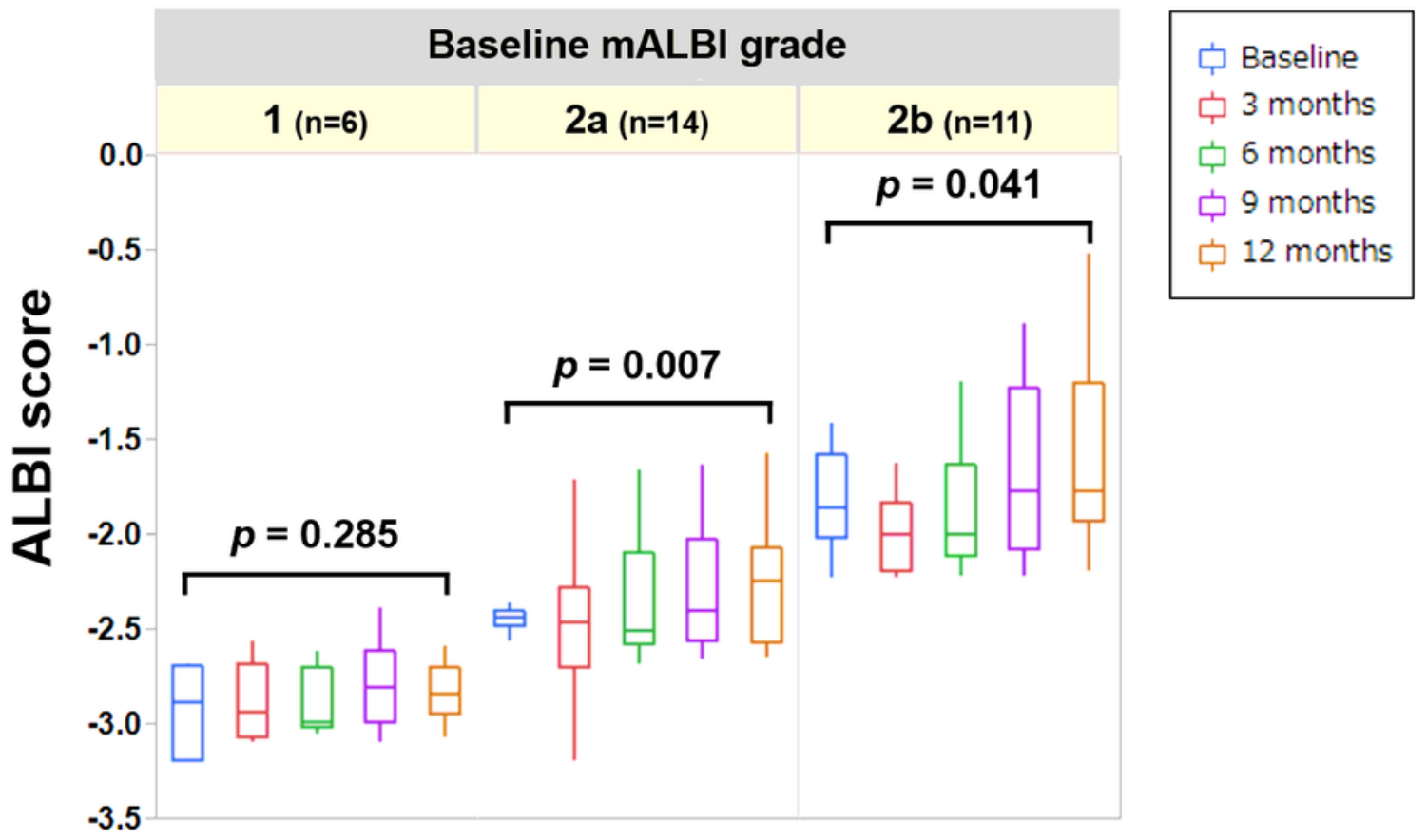

Figure 2 
Subgroup analysis of the longitudinal changes of ALBI score after SBRT. The whole cohort was divided into three subgroups according to the baseline modified ALBI (mALBI) grade.

Figure. $3 \mathrm{~A}$

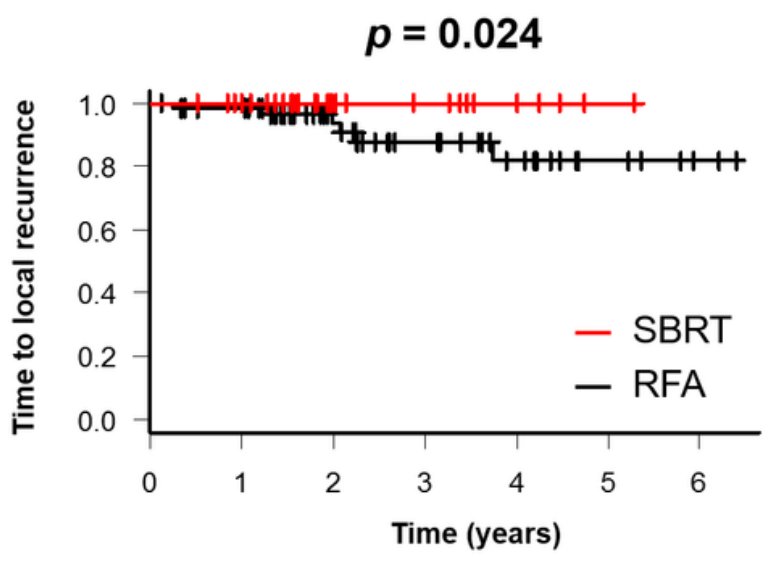

Figure. 3B

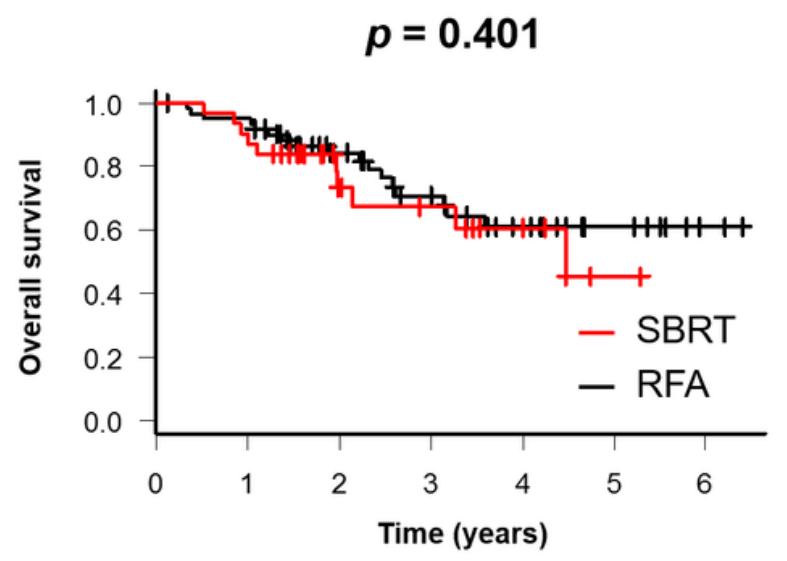

\section{Figure 3}

Subgroup analysis of the longitudinal changes of ALBI score after SBRT. The whole cohort was divided into three subgroups according to the baseline modified ALBI (mALBI) grade. 